American Journal of Neuroscience 3 (1): 32-38, 2012

ISSN 1948-9900

(C) 2012 Science Publications

\title{
The Glia Connection of the Glutamate/Glutamine Shuttle
}

\author{
${ }^{1}$ Angelina Rodriguez and ${ }^{2}$ Arturo Ortega \\ ${ }^{1}$ Faculty of Chemistry, Universidad, \\ Autonoma de Queretaro, Centro Universitario, Queretaro, Mexico \\ ${ }^{2}$ Departament of Genetics and Molecular Biology, \\ Cinvestav-IPN, Apartado Postal 14-740, Mexico DF 07360, Mexico
}

\begin{abstract}
Problem statement: Glia cells outnumber neurons but their role in synaptic transmission is still matter of debate. The recycling of Glutamate, the main excitatory neurotransmitter, carried out by the glutamate/glutamine shuttle, requires the involvement of glia, suggesting their involvement in neurotransmission. Approach: This review focuses on novel functions of glia proteins involved in this cycle. Results: An activity-dependent interaction of glial glutamate transporters, the $\mathrm{Na}^{+/ \mathrm{K}+}$ ATPase, the glutamine and glucose transporters might support glutamatergic neurotransmission. Conclusion: Glia cells that surround glutamatergic contacts, respond to synaptic activity and modify accordingly, the amount and function of the proteins involved in their interaction with neurons thus assuring a synaptic transmission.
\end{abstract}

Key words: Glia cells, glutamate/glutamine shuttle, glutamate transporters, glutamine transporters, glia/neuronal coupling

\section{INTRODUCTION}

Regarded as passive elements, the functions of glia cells are nowadays re-evaluated. The description and characterization of neurotransmitter receptors expressed in their plasma membranes, attracted the attention of a number or researchers which over the years have contributed to the study of glia physiology in the context of neurotransmission (Parpura and Verkhratsky, 2012). The concept of the tripartite synapse: pre-synapsis, postsynapsis and glial cells, often referred as non-existent, has its better example in glutamatergic as well as gabaergic synapses, the reason is simple, the turnover of both of these transmitters require the synthesis of the neutral amino acid glutamine by glutamine synthetase, a glial enzyme (Albrecht et al., 2010). A review of the most recent findings concerning the so-called glutamate/glutamine shuttle that provide strong support the concept of the tripartite synapse follows.

Glial cells: The contribution of neurons to brain function has been widely evaluated and actually gave birth to The Neurosciences. Nevertheless, glia cells outnumber neurons approximately by a factor of ten. In the Central Nervous System (CNS) different types of glial cells regulate aspects like architecture, function and plasticity. Glia cells are divided into two main types: microglia and macroglia. The three main functional classes of macroglial cells in the CNS are: Ependymoglia, myelinating glia and astrocytes. Ependymoglia includes radial glia from the retina and the cerebellum (Müller and Bergmann cells). Myelin forming cells include oligodendroglia in the CNS and Schwann cells in the peripheral nervous system. Astrocytes have an important role in brain development and function (Perea and Araque, 2010). For example, astrocytes release heparin sulphate proteoglycans and by these means promote the formation of excitatory synapses (Allen et al., 2012). Therefore it is clear that these cells are fundamental for neuronal survival and have been usually associated with support and replenishment of metabolic substrates. Despite of this, recent findings have called the attention to the involvement of glia in synaptic transactions throughout the CNS (Eroglu and Barres, 2010). Astrocytes, through a battery of neurotransmitter receptors and transporters present in their plasma membrane, are capable to release neuroactive molecules (glutamate, D-serine, ATP, glutamine, GABA,) that bind to pre and postsynaptic receptors. Additionally, classical transmitters evoke a transient increase in $\left[\mathrm{Ca}^{2+}\right]$ intracellular levels in cultured astrocytes, or in brain slices, displaying a rough form of excitability, although astrocytes are considered as non-excitable cells, since

Corresponding Author: Arturo Ortega, Departament of Genetics and Molecular Biology, Cinvestav-IPN, Apartado Postal 14-740, Mexico DF 07360, Mexico Tel: 525557473769 
they are not capable to generate action potentials (Perea and Araque, 2010). These and other studies led to the proposal of the so-called "tripartite synapse" in which the astrocyte listens to synaptic activity and provides a feedback modulation of the strength of the synaptic connection (Haydon and Carmignoto, 2006). The synaptic control of the astrocyte $\mathrm{Ca}^{2+}$ signal is based in spatially restricted areas called "microdomains" of the astrocytic processes (Grosche et al., 1999). Ultrastructural studies have shown the presence of small synaptic-like vesicles located in close proximity to synapses, apposed either to presynaptic and postsynaptic elements that are thought to contain the mentioned neuroative substances (Jourdain et al., 2007). Glutamate was one of the first neuroactive molecules known to be released by astrocytes that exert an effect on neural excitability.

Glial glutamate receptors: Glutamate receptors have been classified in terms of their signalling strategy in ionotropic (iGluRs) and metabotropic (mGluRs) receptors. The iGluRs are ligand-gated ion channels that are activated by selective agonists: N-methyl-Daspartate (NMDA), $\quad \alpha$-Amino-3-hydroxy-5Methylisoxazole-4-Propionate (AMPA) and Kainate (KA) each one of them was representing a family of homo or heteroligomer receptors (Gasic and Heinemann, 1992). Metabotropic receptors are Gprotein coupled receptors that are divided based on their primary structure into group I, group II and group III and are activated preferentially by quisqualate (Quis), 1-Amino-4, 5-Ciclopentane-trans-1, 3-Dicarboxylate (tACPD) and L-2-amino-phosphonobutanoate (L-AP-4) (Pin and Duvoisin, 1995). Glia cells of different brain structures express both types of receptors, of particular interest is to mention that these receptors have been extensively studied in glial cells that surround glutamatergic synapses like cerebellar Bergmann glia and retinal Müller glia cells (Bellamy, 2006). Bergmann glia cells display a glutamate-dependent continuous dialogue with Purkinje and granules cells, through $\mathrm{Ca}^{2+}$-permeable AMPA receptors. A series of elegant experiments transducing the $\mathrm{Na}^{+}$-determinant AMPA subunit, GluR2 into Bergmann glia cells, modifies its architecture and its physical contacts with Purkinje cells (Iino et al., 2001). It also should be noted that neuronal stimulation elicits glutamate-dependent changes in glial membrane potential in a number of preparations and that these electrical responses are carried out not only by GluRs but also by the $\mathrm{Na}^{+}$dependent glutamate transporters (see below). In any event, glial GluRs, like their neuronal counterpart, are linked to gene expression regulation both at the transcriptional as well as the translational level (Gallo and Ghiani, 2000; Rosas et al., 2007). In this context, it is pertinent to emphasize that among the genes that are regulated by glial GluRs are the glutamate transporters. It is quite possible then that glutamate released activates neuronal and glial receptors, modifying gene expression patterns in both cell types and that among the target genes, those involved in glia/neuronal interactions are represented.

Glial glutamate transporters: Glial glutamate transporters are important for the removal of this neurotransmitter from the synaptic cleft. Five glutamate transporters have been characterized: the $\mathrm{Na}^{+}-$ dependent glutamate/aspartate transporter (GLAST/EAAT-1), the glutamate transporter 1 (Glt1/EAAT-2), the excitatory amino acid carrier 1 (EAAC1/EAAT3), the Excitatory Amino Acid Transporter 4 (EAAT-4) and the Excitatory Amino Acid Transporter 5 (EAAT-5) (Danbolt, 2001). GLAST and Glt-1 are expressed mainly in glia cells while the other three transporters are expressed in neurons. The importance of glial glutamate transporters in pathological scenarios has been deduced from the knock out studies, that demonstrated an elevation of glutamate extracellular levels, neurodegeneration and progressive paralysis (Rothstein et al., 1996). The bulk of glutamate transport in the cerebellum is carried out by GLAST, whereas in the other brain areas it is accomplished by Glt-1. Therefore, glial glutamate transporters are key elements in the prevention of over-stimulation of glutamate receptors, a process that triggers neuroplastic changes and excitotoxic cascades in several pathological conditions (Trotti et al., 2001). In this sense, it has been postulated that disruption of glial glutamate transport affects the time course, fidelity and modulation of excitatory transmission.

The mRNA levels of GLAST and Glt-1 have been investigated during development and in pure glial preparations. At the early stages of development both mRNAs are present in significant amounts, especially at the time of gliogenesis (mouse E15-E19). At birth, GLAST is present in abundance while Glt-1 is barely detectable. In fact, GLAST has been considered as a glia lineage marker (Kriegstein and Alvarez-Buylla, 2009). Glial glutamate transport is regulated in the short and the long term. Short-term regulation includes cellsurface expression and post translational modifications like phosphorylation, ubiquitination and/or acetylation that in one way or another modifying transporter expression at the plasma membrane (Robinson, 2006). 
Long-term regulation includes transcriptional as well as translational control (Lopez-Bayghen and Ortega, 2011). Diverse stimulus are known to affect glial glutamate transporters function, among them glutamate is the most important. It has been shown that glutamate regulates GLAST at the short and long-term, in both cases the net result is a decrease in glutamate uptake activity, albeit the molecular mechanisms are different. In the short-term, glutamate decreases the amount of plasma membrane transporters by interfering with the traffic of the protein to and from the membrane. This is a transporter-dependent effect (Gonzalez and Ortega, 2000). In contrast, in the long-term glutamate, acting through its receptors, down regulates the transcription of the GLAST gene (Rosas et al., 2007). It should be mentioned that this regulation takes place in the cerebellum, while an opposite effect has been recorded for long-term effects of glutamate in the cerebral cortex, where an increase in glast has been detected (Gegelashvilli et al., 2000).

Recently, the role of transporters as signalling entities has began to emerge and glial glutamate transporters are no exception. A transporter dependent increase in p42/44 mitogen kinase activity and in activity of the Mammalian Target of Rapamycin (MTOR) has also been reported (Martinez-Lozada et al., 2011). Furthermore, a signalling complex containing GLAST and the $\mathrm{Na}^{+} / \mathrm{K}^{+}$ATPase has also been described (Gegelashvilli et al., 2007; Rose et al., 2009).

Glial glutamine transporters: Glutamine is the most abundant amino acid in plasma and in the brain extracellular space (Hamberger and Nystrom, 1984). It is the main precursor of glutamate and GABA (Hamberger et al., 1979; Paulsen et al., 1988). Glutamine uptake activity in the brain presents a particular challenge since it is substrate of multiple transporter proteins that also move other neutral amino acids (Barker and Ellory, 1990). The molecular and functional properties of the various amino acid transport systems are characterized by their overlapping substrate specificities, generally low substrate affinities and widespread cellular distribution (Collarini and Oxender, 1987; Broer and Brookes, 2001). The solute carrier families in mammalian cells are the solute carriers SLC1, SLC7 and SLC38 (Hediger et al., 2004; Kanai and Hediger, 2004). SLC1 are $\mathrm{Na}^{+}$dependent neutral amino acid exchange proteins that prefer the substrates alanine, serine and cysteine and therefore are known as the ASC family. Two members ASCT1 and ASCT2 comprise this family and function as exchangers capable to uptake or release amino acids (Christensen et al., 1967). ASCT1 is the most abundant isoform in the brain, despite of this; glutamine is a poor substrate for this transporter (Zerangue and Kavanaugh, 1996). In contrast, ASCT2 transports glutamine and is expressed in mainly in primary cultures of rat astrocytes but also at lower levels in adult and embryonic brain (Broer et al., 1999). The evidence for a neuronal ASCT2 activity is weak (Su et al., 1997) although recent data demonstrates ASCT2 immunoreactivity in cerebellar Purkinje cell bodies and dendrites (Giddon et al., 2009).

SLC7 is a $\mathrm{Na}^{+}$independent transporter and its preference for leucine, gave rise to its cognate name as System L. This family was originally described in kidney tubule cells and includes the heterodimeric transporters LAT1 and LAT2. A low-affinity, highcapacity glutamine uptake activity was described by this system in astrocytes and neurons (Nagaraja and Brookes, 1996; Su et al., 1997). Functional studies suggest a minor role for system L-mediated glutamine transport in astrocytes, contributing with approximately $10 \%$ of the total uptake (Broer and Brookes, 2001; Sidoryk-Wegrzynowicz et al., 2011).

The SCL38 family corresponds to a $\mathrm{Na}^{+}$-dependent neutral amino acid transporters that are divided in two different systems, known as system A and system $\mathrm{N}$, based on the ability of the former to transport alanine and the capacity of the latter to transport amino acids with Nitrogen in its $\mathrm{R}$ group. Both systems respond to hormonal regulation and their function and expression are associated with volume regulation, nutrition and metabolism (Rennie et al., 1998; Haussinger, 1990). System A members include Sodium-Dependent Neutral Amino acid Transporter 1 (SNAT1); SNAT2 and SNAT4, all of them transport small zwitterionic amino acids and are pH sensitive (Albers et al., 2001; Chaudhry et al., 2002). SNAT1 protein expression is confined to the brain, retina, placenta and heart. Within the CNS, SNAT1 its expression is restricted to neurons (Mackenzie et al., 2003; Mackenzie and Erickson, 2004) as does that of SNAT2, both transporters are particularly associated to glutamatergic neurons (Gonzalez-Gonzalez et al., 2005; Melone et al., 2006). In contrast, SNAT4 is expressed in perivenous hepatocyates ( $\mathrm{Gu}$ et al., 2003), glutamine is not the preferred substrate and it also transports cationic amino acids independent of $\mathrm{Na}+$ (Sugawara et al., 2000).

System N has two isoforms: SNAT3 and SNAT5, both coupled to $\mathrm{Na}^{+}$and $\mathrm{H}^{+}$gradients. These transporters are extremely important since these proteins are glutamine carriers capable to mediate glutamine influx and efflux (Baird et al., 2004; Broer et al., 2002; Boulland et al., 2003). It is not surprising that 
its expression in the brain is largely confined to astrocytes (Chaudhry et al., 1999; Boulland et al., 2003).

Glutamate/glutamine shuttle: Glutamine is required as a precursor for other amino acids, protein synthesis and metabolism processes. Particularly, glutamine has an important role in kidney ammoniagenesisis (Wadoux and Welbourne, 1975), driving the urea cycle in the liver nitrogen metabolism (Haussinger, 1990) and in the glutamate/glutamine cycle in the brain (Rothstein and Tabakoff, 1984). This shuttle provides an interesting model to understand the cooperative function of different transporters that interact in synaptic transmission and the release of neurotransmitters. In glutamatergic synapses, the Vesicular Glutamate Transporters (VGLUT) (Takamori et al., 2000) located in the presynaptic neuron; charge the synaptic vesicles with the glutamate produced by the hydrolysis of glutamine by Glutaminase. Synaptic vesicles contain large amounts of glutamate and release it by exocytosis to the synaptic cleft, where it interacts with glutamatergic receptors and transporters. Glutamate is cleared from the synaptic space by the glial glutamate transporters (GLAST and Glt-1) (Schousboe, 1981) where Glutamine Synthetase (GS) converts it to glutamine. Glia cells, using system $\mathrm{N}$ transporters (SNAT3 and SNAT5) mediate the efflux of glutamine to the extracellular milieu (Chaudhry et al., 2002), to be taken up by neurons by system A transporters (SNAT1 and SNAT2) (Varoqui et al., 2000) completing the cycle (Daikhin and Yudkoff, 2000).

It should be note, however that neurons do not depend exclusively on the astrocytic shuttle cycle for the replenishment of glutamate. The glutamate uptake via EAAC1/EAAT3 transporter and the glutamate synthesis from the tricarboxylic cycle, are two potential glutamatergic sources involved in the neuron replenishment of glutamate (Hertz et al., 2000; Broer and Brookes, 2001). The dysfunctionality of any of the steps in the cycle is associated whit a variety of neurological disorders and conditions (Cruz and Cerdan, 1999).

A tripartite synapse: The Glia connection: Glutamatergic synapses are unique structures in which the actual contribution of glial cells to neurotransmission has been reported (Iino et al., 2001; Lopez-Bayghen et al., 2007; Uwechue et al., 2012). It is tempting to speculate that once the presynaptic terminal is stimulated and glutamate released, the amino acid is taken up avidly by the glial glutamate transporters (either GLAST in cerebellum or Glt-1 in most of the other brain structures) resulting in a net $\mathrm{Na}^{+}$influx.

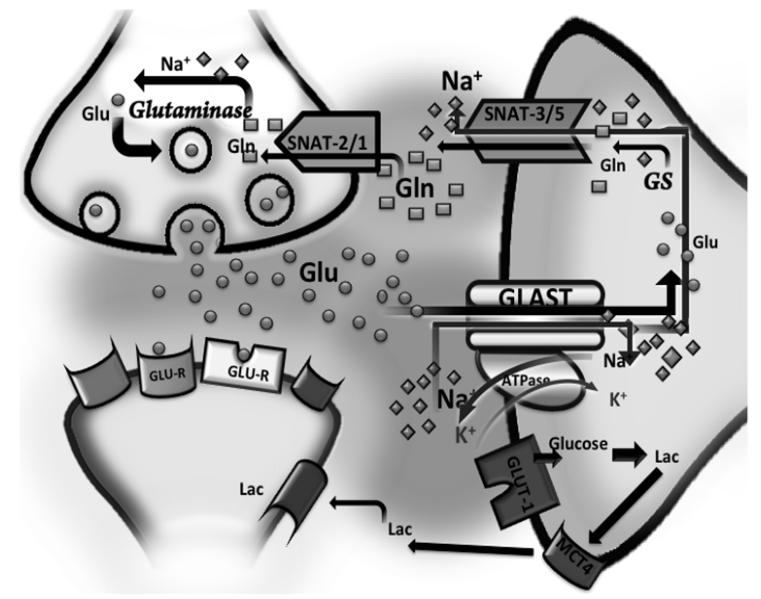

Fig. 1: Summary of the molecular transactions involved in the glia/neuronal interactions upon glutamatergic stimulation. Glutamate released from the presynaptic terminal reaches postsynaptic and glial glutamate receptors and transporters (see text for details). Abbreviations: GluRs, glutamate receptors, GLAST, $\mathrm{Na}^{+}$-dependent glutamate/aspartate transporter. Glut-1, glucose transporter 1. GS, glutamine synthetase, Lac, lactate, MCT-4, monocarboxylate transporter 4. SNAT, sodiumdependant neutral amino acid transporter

Increased intracellular $\mathrm{Na}^{+}$triggers glucose uptake (Maekawa et al., 2006) and glutamine release (Uwechue et al., 2012) to be finally pumped out through that transporter-associated $\mathrm{Na}^{+} / \mathrm{K}^{+}$ATPase (Rose et al., 2009). A sustained glutamatergic activity would envisage a reduction in ATP levels, which would shut down transiently protein synthesis (Barrera et al., 2010). The properties thus far described for the glial proteins involved in this hypothesis support this elegant and exquisite coupling. A schematic diagram of our interpretation of the role of glial cells in the tripartite synapse is depicted in Fig. 1.

\section{CONCLUSION}

Glial cells participate actively in the formation and function of glutamatergic synapses. The glial protein repertoire is constantly responding to synaptic activity and therefore these cells should be considered as an integral part of the synapses.

\section{ACKNOWLEDGMENT}

The study in our laboratories is supported by grants from PROMEP/SEP to A.R (UAQ-PT-118) and CONACyT-Mexico to A.O. (79502). 
Am. J. Neuroscience 3 (1): 32-38, 2012

\section{REFERENCES}

Albers, A., A. Broer, C.A. Wagner, I, Setiawan and P.A. Lamg et al., 2001. $\mathrm{Na}^{+}$transport by the neural glutamine transporter ATA1. Pflugers Arch, 443: 92-101. PMID: 11692272

Albrecht, J., M. Sideoryk-Wergrzynowicz, M. Zielinska and M. Aschner, 2010. Roles of glutamine in neurotransmission. Neuron. Glia. Biol., 6: 263-276. PMID: 22018046

Allen, J.A., M.L. Bennet, L.C. Foo., G.X. Wang and C. Chakraborty et al., 2012. Astrocyte glypicans 4 and 6 promote formation of excitatory synapses via GluA1 AMPA receptors. Nature, 486: 410-414. DOI: $10.1038 /$ nature 11059

Baird, F.E., K.J. Beattie, A.R. Hyde, V. Ganapathy and M.J. Rennie et al., 2004. Bidirectional substrate fluxes through the system N (SNAT5) glutamine transporter may determine net glutamine flux in rat liver. J. Physiol., 559: 367-381. PMID: 15218073

Barker, G.A. and J.C. Ellory, 1990. The identification of neutral amino acid transport systems. Exp. Physiol., 75: 3-26.

Barrera, I., M.A. Flores-Mendez, L.C. HernandezKelly, L. Cid and M. Huerta et al., 2010. Glutamate regulates eEF1A phosphorylation and ribosomal transit time in Bergmann glial cells. Neurochem. Int. 57: 795-803. PMID: 20817065

Bellamy, T.C., 2006. Interactions between Purkinje neurones and Bergmann glia. Cerebellum, 5: 116126. PMID: 16818386

Broer, A., N. Brookes, V. Ganapathy, K.D. Dimmer, C.A. Wagner and F. Lang et al., 1999. The astroglial ASCT2 amino acid transporter as a mediator of glutamine efflux. J. Neurochem, 73: 2184-2194. $\quad$ DOI: $\quad 10.1046 / j .1471-$ 4159.1999.02184.x

Broer, S. and N. Brookes, 2001. Transfer of glutamine between astrocytes and neurons. J. Neurochem, 77: 705-719. PMID: 11331400

Broer, A., A. Albers, I. Setiawan, R.H. Edwards and F.A. Chaudhry et al., 2002. Regulation of the glutamine transporter SN1 by extracellular $\mathrm{pH}$ and intracellular sodium ions. J. Physiol., 539: 3-14. PMID: 11850497

Boulland, J.L., A. Rafiki, L.M. Levy, J. StormMathisen and F.A. Chaudhry, 2003. Highly differential expression of SN1, a bidirectional glutamine transporter, in astroglia and endothelium in the developing rat brain. Glia, 41: 260-275. PMID: 12528181
Christensen, H.N., M. Liang and E.G. Archer, 1967. A distinct Na+-requiring transport system for alanine, serine, cysteine and similar amino acids. J. Biol. Chem., 242: 5237-5246. PMID: 6070848

Chaudhry, F.A., R.J. Reimer, D. Krizaj, D. Barber and J. Storm-Mathisen et al., 1999. Molecular analysis of system $\mathrm{N}$ suggests novel physiological roles in nitrogen metabolism and synaptic transmission. Cell, 99: 769-780. PMID: 10619430

Chaudhry, F.A., D. Schmitz, R.J. Reimer, P. Larsson and A.T. Gray et al., 2002. Glutamine uptake by neurons: Interaction of protons with system a transporters. J. Neurosci., 22: 62-72. PMID: 11756489

Collarini, E.J. and D.L. Oxender, 1987. Mechanisms of transport of amino acids across membranes. Ann. Rev. Nutr., 7: 75-90. PMID: 3300747

Cruz, F. and S. Cerdan. 1999. Quantitative 13C NMR studies of metabolic compartmentation in the adult mammalian brain. NMR Biomed., 12: 451-462. PMID: 10654292

Danbolt, N.C., 2001. Glutamate uptake. Prog. Neurobiol., 65: 1-105. PMID: 11369436

Daikhin, Y. and M. Yudkoff, 2000. Compartmentation of brain glutamate metabolism in neurons and glia. J. Nutr., 130: 1026S-1031S. PMID: 10736375

Eroglu, C. and B.A. Barres, 2010. Regulation of synaptic connectivity by glia. Nature, 468: 223231. PMID: 21068831

Gallo, V. and C.A. Ghiani, 2000. Glutamate receptors in glia: New cells, new inputs and new functions. Trends. Pharmacol. Sci., 21: 252-258. PMID: 10871893

Gasic, G.P. and S. Heinemann, 1992. Determinants of the calcium permeation of ligand-gated cation channels. Curr. Opin. Cell Biol., 4: 670-677. PMID 1419048

Gegelashvilli, G., Y. Dehnes, N.C. Danbolt and A. Schousboe, 2000. The high-affinity glutamate transporters GLT1, GLAST and EAAT4 are regulated via different signalling mechanisms. Int., 37: 163-170. PMID: 10812201

Gegelashvilli, M., A. Rodriguez-Kern, L. Sung, K. Shimamoto and G. Gegelashvilli, 2007. Glutamate transporter GLAST/EAAT1 directs cell surface expression of FXYD2/gamma subunit of $\mathrm{Na}, \mathrm{K}$ ATPase in human fetal astrocytes. Neurochem. Int., 50: 916-920. PMID: 17316900

Giddon, C.M, Z. Shao, J.L. Maistre and C.M. anderson, 2009. Cellular distribution of the neutral amino acid transporter subtype ASCT2 in mouse brain. J. Neurochem., 108: 372-383. PMID: 19012749 
Gonzalez-Gonzalez, I.M., B. Cubelos, C. Gimenez and F. Zafra, 2005. Immunohistochemical localization of the amino acid transporter SNAT2 in the rat brain. Neuroscience, 130: 61-73. PMID: 15561425

Gonzalez, M.I. and A. Ortega, 2000. Regulation of high-affinity glutamate uptake activity in Bergmann glia cells by glutamate. Brain Res., 866: 73-81. PMID: 10825482

Grosche, J., V. Matyash, T. Moller, A. Verkhratsky and A. Reichenbach et al., 1999. Microdomains for neuron-glia interaction: Parallel fiber signaling to Bergmann glial cells. Nat. Neurosci., 2: 139-143. PMID: 10195197

Gu, S., P. Langlais, F. Liu and J.X. Jiang, 2003. Mouse system-N amino acid transporter, mNAT3, expressed in hepatocytes and regulated by insulinactivated and phosphoinositide 3-kinase-dependent signalling. Biochem. J., 371: 721-731. PMID: 12537539

Hamberger, A., G.H. Chiang, E. Sandoval and C.W. Cotman, 1979. Glutamate as a CNS transmitter. II. Regulation of synthesis in the releasable pool. Brain. Res., 168: 513-530. PMID: 435981

Hamberger, A. and B. Nystrom, 1984. Extra- and intracellular amino acids in the hippocampus during development of hepatic encephalopathy. Neurochem. Res., 9: 1181-1192. PMID: 6504234

Haussinger, D., 1990. Shifting of hepatic glutamine balance from uptake to output: Role of liver cell heterogeneity. Nutrition, 6: 412-413. PMID: 2134567

Haydon, P.G. and G. Carmignoto, 2006. Astrocyte control of synaptic transmission and neurovascular coupling. Physiol. Rev., 86: 1009-1031. PMID: 16816144

Hediger, M.A., M.F. Romero, J.B. Peng, A. Rolfs and H. Takanaga et al., 2004. The ABCs of solute carriers: physiological, pathological and therapeutic implications of human membrane transport proteinsIntroduction. Pflugers. Arch, 447: 465-468. PMID: 14624363

Hertz, L., A.C. Yu, G. Kala and A. Schousboe, 2000. Neuronal-astrocytic and cytosolic-mitochondrial metabolite trafficking during brain activation, hyperammonemia and energy deprivation. Neurochem. Int., 37: 83-102. PMID: 10812194

Iino, M.., K. Goto, W. Kakegawa, H. Okado and M. Sudo et al., 2001. Glia-synapse interaction through Ca2+-permeable AMPA receptors in Bergmann glia. Science, 292: 926-929. PMID: 11340205
Jourdain, P., L.H. Bergersen, K. Bhaukaurally., P. Bezzi and M. Santello et al., 2007. Glutamate exocytosis from astrocytes controls synaptic strength. Nat. Neurosci., 10: 331-339. PMID: 17310248

Kanai, Y. and M.A. Hediger, 2004. The glutamate/neutral amino acid transporter family SLC1: molecular, physiological and pharmacological aspects. Pflugers. Arch, 447: 469479. PMID: 14530974

Kriegstein, A. and A. Alvarez-Buylla, 2009. The glial nature of embryonic and adult neural stem cells. Annu. Rev. Neurosci., 32: 149-184. DOI: 10.1146/aannnurev.neuro.051508.135600

Lopez-Bayghen, E., S. Rosas, F. Castelan and A. Ortega, 2007. Cerebellar bergmann glia: an important model to study neuron-glia interactions. Neuron. Glia. Biol., 3: 155-167. PMID: 18634572

Lopez-Bayghen, E. and A. Ortega, 2011. Glial glutamate transporters: New actors in brain signalling. IUBMB Life, 63: 816-823. DOI: 10.1002/iub.536

Mackenzie, B., M.K. Schafer, J.D. Erickson, M.A. Hediger and E. Weihe et al., 2003. Functional properties and cellular distribution of the system a glutamine transporter SNAT1 support specialized roles in central neurons. J. Biol. Chem., 278: 23720-23730. PMID: 12684517

Mackenzie, B. and J.D. Erickson, 2004. Sodiumcoupled neutral amino acid (System N/A) transporters of the SLC38 gene family. Pflugers. Arch, 447: 784-795. PMID: 12845534

Maekawa, F., K. Minehira, K. Kadomatu and L. Pellerin, 2006. Basal and stimulated lactate fluxes in primary cultures of astrocytes are differentially controlled by distinct proteins. J. Neurochem., 107: 789-798. PMID 18761711

Martinez-Lozada, Z., L.C. Hernandez-Kelly, J. Aguilera, E. Lopez-Bayghen and A. Ortega, 2011. Signaling through EAAT-1/GLAST in cultured Bergmann glia cells. Neurochem. Int., 59: 871-879. PMID: 21856347

Melone, M., H. Varoqui, J.D. Erickson and F. Conti, 2006. Localization of the $\mathrm{Na}(+)$-coupled neutral amino acid transporter 2 in the cerebral cortex. Neuroscience, 140: 281-292. PMID: 16616430

Nagaraja, T.N. and N. Brookes, 1996. Glutamine transport in mouse cerebral astrocytes. J. Neurochem., 66: 1665-1674. PMID: 8627324

Parpura, V. and A. Verkhratsky, 2012. Neuroglia at the crossroads of homoeostasis, metabolism and signalling: Evolution of the concept. ASN Neuro., 4: 201-205. PMID: 22455879 
Paulsen, R.E., E. Odden and F. Fonnum, 1988. Importance of glutamine for gamma-aminobutyric acid synthesis in rat neostriatum in vivo. $\mathrm{J}$. Neurochem., 51: 1294-1299. PMID: 2901465

Perea, G. and A. Araque, 2010. GLIA modulates synaptic transmission. Brain Res. Rev., 63: 93-102. PMID: 19896978

Pin, J.P. and R. Duvoisin, 1995. The metabotropic glutamate receptors: Structure and functions. Neuropharmacol, 34: 1-26. PMID: 7623957

Rennie, M.J., S.Y. Low, P.M. Taylor, S.E. Khogali and P.C. Yao, 1998. Amino acid transport during muscle contraction and its relevance to exercise. Adv. Exp. Med. Biol., 441: 299-305. PMID: 9781335

Rosas, S., M.A. Vargas, E. Lopez-Bayghen and A. Ortega, 2007. Glutamate-dependent transcriptional regulation of GLAST/EAAT1: A role for YY1. J. Neurochem., 101: 1134-1144. PMID: 17394550

Robinson, M.B., 2006. Acute regulation of sodiumdependent glutamate transporters: A focus on constitutive and regulated trafficking. Hand. Exp. Pharmacol., 2006: 251-275. PMID: 16722240

Rose, E.M., J.C. Koo, J.E. Antfflick, S.M. Ahmed and S. Angers et al., 2009. Glutamate transporter coupling to Na,K-ATPase. J. Neurosci., 24: 81438155. PMID: 19553454

Rothstein, J.D. and B. Tabakoff, 1984. Alteration of striatal glutamate release after glutamine synthetase inhibition. J. Neurochem, 43: 1438-1446. PMID: 6149260

Rothstein, J.D., M. Dykes-Hoberg, C.A. Pardo, L.A. Bristol and R.W. Kumcl et al., 1996. Knockout of glutamate transporters reveals a major role for astroglial transport in excitotoxicity and clearance of glutamate. Neuron, 16: 675-686. PMID: 8785064

Sidoryk-Wegrzynowicz, M., E. Lee, N. Mingwei and M. Aschner, 2011. Disruption of astrocytic glutamine turnover by manganese is mediated by the protein kinase C pathway. Glia, 59: 1732-1743. DOI: $10.1002 /$ glia.21219
Su, T.Z., G.W. Capmbell and D.L. Oxender, 1997. Glutamine transport in cerebellar granule cells in culture. Brain Res., 757: 69-78. PMID: 9200500

Sugawara, M., T. Nakanishi, Y.J. Fei, R.G. Martindale and M.E. Ganapathy et al., 2000. Structure and function of ATA3, a new subtype of amino acid transport system A, primarily expressed in the liver and skeletal muscle. Biochim. Biophys. Acta, 1509: 7-13. PMID: 11118514

Schousboe, A., 1981. Transport and metabolism of glutamate and GABA in neurons are glial cells. Int. Rev. Neurobiol., 22: 1-45. PMID: 6115823

Takamori, S., J.S. Rhee, C. Rosenmund and R. Jahn, 2000. Identification of a vesicular glutamate transporter that defines a glutamatergic phenotype in neurons. Nature, 407: 189-194. PMID: 11001057

Trotti, D., M. Aoki, P. Pasinelli, U.V. Berger and N.C. Danbolt et al., 2001. Amyotrophic lateral sclerosislinked glutamate transporter mutant has impaired glutamate clearance capacity. J. Biol. Chem., 276: 576-582. PMID: 11031254

Uwechue, N.M., M.C. Marx, Q. Chevy and B. Billups, 2012. Activation of glutamat transport evokes rapid glutamine release from preisynaptic astrocytes. J. Physiol., 590: 2317-2331. PMID: 22411007

Varoqui, H., H. Zhu, D. Yao, H. Ming and J.D. Erickson, 2000. Cloning and functional identification of a neuronal glutamine transporter. J. Biol. Chem., 275: 4049-4054. PMID: 10660562

Wadoux, P. and T.C. Welbourne, 1975. Ammoniagenesis: D-glutamyltransferase as a source of ammonia in the rat kidney. Can. J. Biochem., 53: 930-933. PMID: 241474

Zerangue, N. and M.P. Kavanaugh, 1996. ASCT-1 is a neutral amino acid exchanger with chloride channel activity. J. Biol. Chem., 271: 2799127994. PMID: 8910405 